\title{
Features of Varicella Outbreak in Primary Schools in an Integrated Syndromic Surveillance System in Rural China
}

\author{
Changming Zhou*1, Tao Tao', Huijian Cheng², Qi Zhao', Genming Zhao', Xue Li ${ }^{3}$, Shaofa \\ $\mathrm{Nie}^{4}$, Weirong $\mathrm{Yan}^{4,5}$ and Biao $\mathrm{Xu}^{1}$
}

${ }^{1}$ Fudan University, Shanghai, China; ${ }^{2}$ Jiangxi Province Center for Disease Prevention and Control, Nanchang, China; ${ }^{3}$ Future Position X, Gävle,, Sweden; ' ${ }^{4}$ epartment of Epidemiology and Biostatistics, School of Public Health, Tongji Medical College of Huazhong University of Science and Technology, Wuhan, China; ${ }^{5}$ Division of Global Health (IHCAR), Department of Public Health Sciences, Karolinska Institutet, Stockholm, Sweden

\section{Objective}

To describe the features of varicella outbreak in rural primary schools and the impacts of school absenteeism surveillance on early detection of infectious disease outbreak.

\section{Introduction}

Since April 2012, an integrated syndromic surveillance system (ISSC) has been established in health facilities, pharmacies and primary schools in two rural counties of Jiangxi Province, China (1). The objective of ISSC is to integrate syndromic surveillance with case report system for infectious disease to improve the early detection of disease outbreak in rural China. Varicella is a common respiratory infectious disease among children. In most cases, it is mild but it might cause severe complications including, pneumonia, meningitis, even death (2). In this study, varicella related school absenteeism and outpatient visits in health facilities in the surveillance sites of ISSC were collected and analyzed.

\section{Methods}

Sixty-two primary schools from 3 levels (county, township, village) in 2 counties were sampled as surveillance sites for students' absenteeism. Each absenteeism information which includes age, class, gender and reasons for absenteeism was required to be reported to ISSC platform. The health facilities in ISSC were 2 county hospitals, 15 township hospitals and 142 village health stations. Ten symptoms including fever, rash were under surveillance. Patients with any of these symptoms should be reported to the ISSC platform with gender, age, village, onset date and visit date. A varicella outbreak was defined as 5 or more varicella cases in one school within one week by local health authority. A public health emergency was defined as 10 or more varicella cases in one school according to National Public Health Emergency Information Reporting and Management Specifications (3).

\section{Results}

During Jan.1 to Jun. 30 2013, varicella outbreaks were observed in 3 county schools and 1 township school. The cases from school D were confined in one class. In school A, B and C, varicella cases firstly appeared in one class and appeared in next-door classes in 2 days. All the cases emerged within the minimum incubation period of 10 days and all the absenteeism lasted for less than one week. (Table 1)

Of all the 25 reported children in the 4 outbreaks, only one child was captured by hospitals. This 8 -year-old boy visited the county hospital for rash, and was clinically diagnosed as varicella. This case was recorded by both ISSC (health facilities) and China Information System for Diseases Control and Prevention (CISDCP). The rest 24 students sought health care in non-surveillance sites including private clinics and traditional Chinese medicine hospitals.

\section{Conclusions}

Varicella outbreak could be observed in county schools or township schools where the students' density is high. When a varicella outbreak happened, a next-door class could be implicated in several days. In this study, the absenteeism of varicella was shorter than the disease course which could be a potential risk for transmission. Most of the outbreak events could not be captured by syndromic surveillance in hospitals because of limited surveillance sites and wide range of healthcare choices. School absenteeism surveillance would be more sensible to varicella and might be a good supplement for early detection. Findings from this study highlighted the necessity of the integration of CISDCP and syndromic surveillance between primary schools and health care system.

Table 1 General information of the varicella outbreak events in two counties of Jiangxi Province, China

\begin{tabular}{|c|c|c|c|c|c|c|}
\hline SchoolCounty & $\begin{array}{c}\text { Level of } \\
\text { school }\end{array}$ & $\begin{array}{c}\text { Duration } \\
\text { (MM/DD) }\end{array}$ & $\begin{array}{c}\text { Initiating class } \\
\text { (varicella cases) }\end{array}$ & $\begin{array}{c}\text { Affected classes } \\
\text { (varicella cases) }\end{array}$ & $\begin{array}{c}\text { Total absenteeism } \\
\text { for varicella }\end{array}$ \\
\hline A & F & County & $01 / 11-01 / 18$ & $2-1(5)$ & $2-2(1)$ & 6 \\
\hline B & F & County & $05 / 13-05 / 17$ & $4-2(5)$ & $4-3(1)$ & 6 \\
\hline C & F & Township & $06 / 03-06 / 09$ & $1-3(2)$ & $\begin{array}{c}1-1(1) 1-2(1) 2-2(1) \\
3-2(1) 1-4(1)\end{array}$ & 7 \\
\hline D & Y & County & $03 / 11-03 / 15$ & $3-4(6)$ & Noun & 6 \\
\hline
\end{tabular}

\section{Keywords}

Varicella; School absenteeism; Surveillance

\section{Acknowledgments}

EU 7th Framework Program

\section{References}

1.Yan W, Palm L, et al. ISS - an electronic syndromic surveillance system for infectious disease in rural China. PLoS One. 2013 2013-0120;8(4):e62749.

2.Gowin E, Wysocki J, Michalak M. Don't forget how severe varicella can be-complications of varicella in children in a defined Polish population. Int J Infect Dis. 2013 2013-07-01;17(7):e485-9.

3.China MOH. National Public Health Emergency Information Reporting and Management Specification.; 2005.

\section{*Changming Zhou}

E-mail: 12111020001@fudan.edu.cn 